\title{
Laser Processing of Printed Copper Interconnects on Polymer Substrates
}

\author{
Kang Wu, Shuai Hou*, Siyuan Qi*, David A. Hutt, John R. Tyrer, David C. Whalley \\ Wolfson School of Mechanical, Electrical and Manufacturing Engineering \\ Loughborough University \\ Loughborough, LE11 3TU, UK \\ *Present address: Manufacturing Technology Centre, Ansty Park, Coventry, CV7 9JU, UK. \\ K.Wu@lboro.ac.uk, D.A.Hutt@lboro.ac.uk
}

\begin{abstract}
With the increasing demand for integration of electronics embedded within devices there has been a consequent increase in the requirement for the deposition of electrically conductive materials to form connecting tracks on or within nontraditional substrate materials, such as temperature sensitive polymers, that may also have non-planar surfaces. In this work, micron scale copper powder based materials were deposited onto acrylic and glass substrates and then selectively laser processed to form electrically conductive copper tracks. Before deposition, the copper powder was chemically treated to remove the surface oxide and subsequently protected with a self-assembled monolayer coating. The copper was then patterned onto the substrate either as a dry powder confined within pre-formed grooves, or was combined with a binder to be printed as a paste. $\mathrm{A} \mathrm{CO}_{2}$ laser was then used to heat the copper powder in air, leading to tracks that showed good electrical conductivity. At low laser power levels, the tracks appeared largely unchanged from the original material, but showed measureable conductivity. With higher laser power levels the tracks showed evidence of partial melting of the surface layers and further reductions in resistivity, to values approximately 30 times those of bulk copper, were obtained.
\end{abstract}

\section{Introduction}

Fabricating patterns of electrically conductive interconnects has always been an essential part of electronics manufacturing and numerous methods, both additive and subtractive, have been applied in achieving this requirement. However, the majority of today's electronic components are interconnected using printed circuit boards (PCBs) having chemically etched copper tracks. The conventional photolithographic patterning and chemical etching method used to manufacture such PCBs is considered a timeconsuming, expensive and environmentally hazardous approach because of its multiple process steps and subtractive nature that produces many waste streams. As a result, industry is constantly seeking alternative approaches which offer low cost, low waste output and simple processing routes. Coupled with this, there is an increasing demand for electronics to be integrated and embedded within a much wider variety of products to support growth areas such as wearables and the Internet of Things (IoT). The alternative form factors and varied substrate materials, including many temperature sensitive polymers, that these emerging applications demand is further increasing the pressure on manufacturers to develop alternative methods of interconnect fabrication.

Additive manufacturing routes to the fabrication of electrically conductive tracks are an attractive approach, due to their high material usage efficiency and design flexibility. Furthermore, they offer the opportunity to incorporate electronic circuits on or within three dimensional components. Presently, inkjet or screen printing of silver filled inks and pastes is widely used to form conductive tracks on both flat (2D) and curved (3D) substrate surfaces with good reliability (e.g. [1],[2]), but the relatively high cost of silver is restricting their widespread uptake, especially in high volume cost conscious applications. In some cases, the requirement for post processing of these materials at elevated temperatures also makes them incompatible with some polymer substrates, particularly low cost thermoplastics. Copper as an interconnect material, has comparably good conductivity, high resistance to electro-migration and a much lower price when compared to silver and gold. However, mainly due to its high susceptibility to the formation of a non-conductive oxide layer, the use of copper filled inks and pastes is often hindered in applications that involve heating it in open air.

Laser processing within the additive manufacturing industry has been studied for many years. Lasers have been shown to provide well controlled and localized energy sources that may be precisely applied, making them suitable for complex 3D structure fabrication. Various studies have investigated the additive manufacturing of structural components [3],[4], with some completed on copper and copper alloys [5]-[9]. Laser direct writing has also been carried out on a layer of previously inkjet printed copper nanoparticles [10],[11]. However using nanoparticles is often considered expensive due to the material preparation methods, the relatively low metal loading that can be deposited and in some cases the requirement for an inert processing atmosphere. Therefore, in this research, a new approach was investigated for the selective formation of copper interconnects on polymeric substrates by the laser processing of micron scale copper powder pre-treated to enable processing in air.

A key requirement for the effective laser sintering of metal powders, or their consolidation through melting, is that the individual particles are able to join together without intermediate oxide layers preventing them from coming into direct contact. In this study copper micron-scale powder was therefore initially chemically pre-treated to remove the surface oxide and then protected from re-oxidation by the deposition of a thin organic self-assembled monolayer (SAM) on the

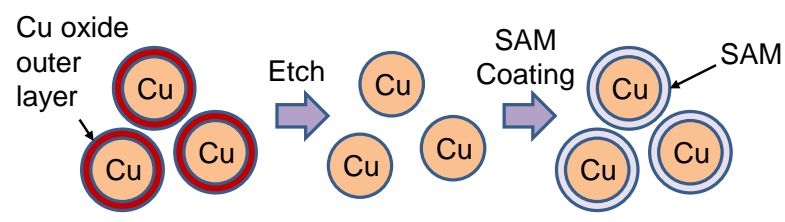

Fig. 1. Schematic view of the copper powder surface modification process. 
surface, as shown in Fig. 1. SAM coatings are usually formed by the adsorption of long chain organic molecules from dilute solutions and create a densely packed film that inhibits the diffusion of oxygen and water vapour to the copper surface, thereby significantly reducing the rate of oxidation [12]. The SAM protective layer used in this study enabled the copper powder to be stored in air at low temperature before use, but would be expected to rapidly degrade during laser heating to facilitate contact between the neighbouring copper particle surfaces. YAG laser systems are often used in laser sintering research due to their good absorption by metals [13],[14]. However, despite the anticipated lower energy absorption by copper of the longer wavelength $\mathrm{CO}_{2}$ laser radiation compared to that of the shorter wavelength YAG [15], the ability of the $\mathrm{CO}_{2}$ system to also readily process many polymeric materials makes it attractive for the future integration of interconnect manufacture with the fabrication of 3D plastic parts. This readily available, low cost and easily controlled laser source was therefore selected for use in this research and a high frequency pulsed $\mathrm{CO}_{2}$ laser was chosen to allow the processing of the pre-treated micron scale copper powder on temperature sensitive polymer substrates.

\section{Experimental}

Micron scale copper powder $(14-25 \mu \mathrm{m}$ particle size, Sigma Aldrich) was used as the raw material in this research. The powder was pre-treated to remove the oxide and then protected from re-oxidation by applying an organic SAM coating. The powder was then stored in a freezer prior to use. To produce tracks on the substrate, the copper was used either as a dry loose powder or prepared as a printable paste (Fig. 2). To deposit the dry powder, a groove was first laser machined in an acrylic substrate to create a receptacle into which the copper powder was spread using a blade (Fig. 2a). Typical groove dimensions were approximately $2 \mathrm{~mm}$ wide, $20 \mathrm{~mm}$ long and $250 \mu \mathrm{m}$ deep, but narrower features were also investigated. As an alternative, the copper powder was combined with a polymer binder material and solvent and then printed using a stencil onto glass or acrylic substrates (Fig. 2b): this produced stripes approximately $2 \mathrm{~mm}$ wide, $20 \mathrm{~mm}$ long and $200 \mu \mathrm{m}$ thick.

A $100 \mathrm{~W}$ water cooled, pulsed $\mathrm{CO}_{2}$ laser (Firestar ti 100 series from Synrad) was used as the energy source to process the copper tracks and to machine the grooves in the acrylic substrate. The laser delivery system is presented in Fig. 2c. The laser scanning pattern was achieved by using Winmark software to control two mirrors that adjusted the optical path based on software commands. Samples were fixed on a horizontal working platform at the focus position of the laser. The experiments presented here were conducted in air, but the effect of using an inert atmosphere around the sample was also investigated in other work. For these experiments, the power of the laser delivered at the sample was typically in the range of 15 to $55 \mathrm{~W}$ and the values stated here showed a typical variation of $\pm 3 \%$. The nominal laser spot diameter was $0.3 \mathrm{~mm}$ and the speed of translation of the spot across the surface was adjusted between 200 and $600 \mathrm{~mm} \mathrm{~s}^{-1}$. As the deposited stripes of copper powder were wider than the beam size, it was found necessary to raster the beam across the stripe in order to process the whole surface area. Therefore, in addition to the laser power and speed of movement of the laser

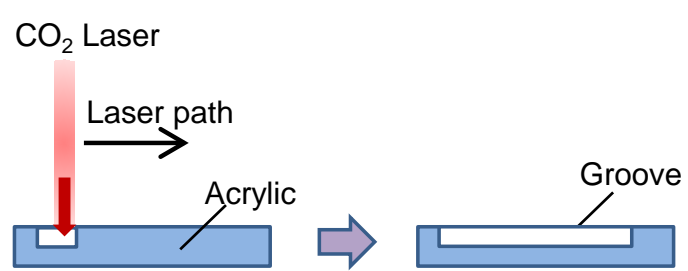

Laser machining of acrylic substrate

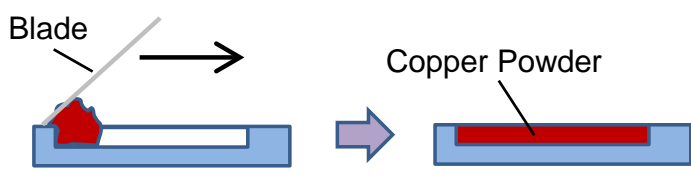

Copper powder filling of the groove

(a)

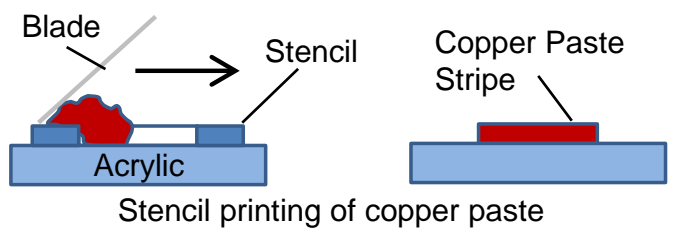

(b)

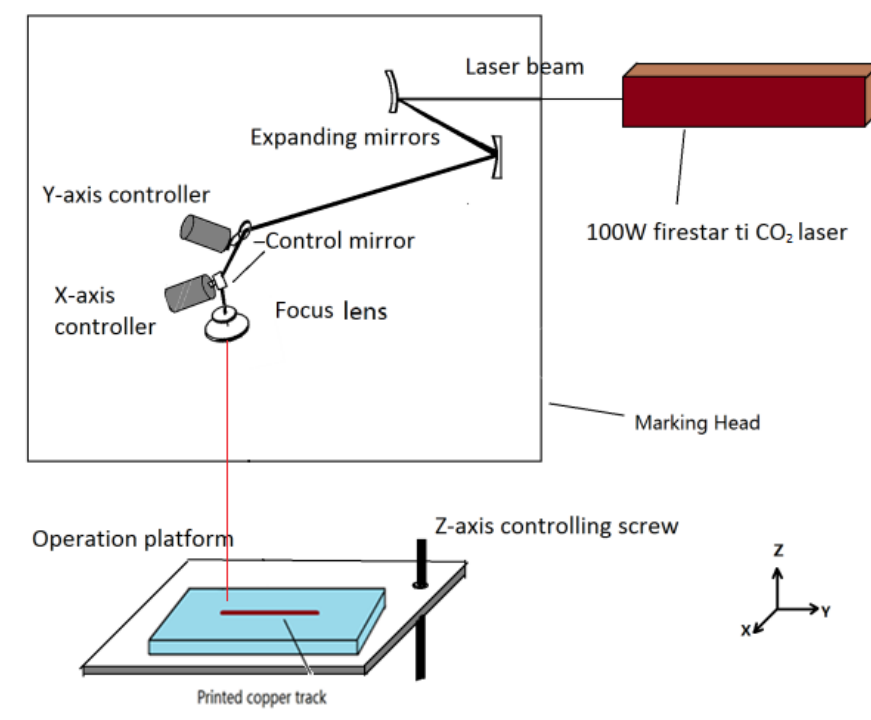

(c)

Fig. 2. Overview of the copper deposition and laser processing methods (a) powder deposition within a groove laser machined in acrylic; (b) stencil printing of copper paste; (c) optical arrangement for laser processing.

spot, the effect of the laser path direction and spacing between the laser passes was also investigated by varying them along and across the printed stripes. Furthermore, in some cases the beam was passed multiple times over the same area to be processed.

Track morphology was characterized using scanning electron microscopy. The resistance of the tracks was measured using a four point probe system and converted to resistivity based on the thickness and width of the tracks and the length over which the measurement was taken. X-ray Photoelectron Spectroscopy (XPS) was also used to investigate the surface condition of the copper powder. 


\section{Results and Discussion}

\section{Copper Powder Characterization and Printing}

The copper powder was characterized using XPS to determine the level of oxidation present on the surface. Fig. 3 shows a typical survey scan of the powder after surface modification and short term storage in air within a freezer. In addition to the clear peaks due to copper, peaks associated with carbon and sulphur, the constituents of the SAM coating, are present. The SAM coating used in these trials did not contain any oxygen species and therefore the oxygen peak in the spectrum at around $532 \mathrm{eV}$ is very small, indicating a low level of oxide in the material, despite its exposure to air.

Fig. 4 shows SEM images of the copper stripes formed by printing the paste and allowing it to dry. The individual particles in the paste can be clearly seen, but between the particles there is no clear evidence of the binder. This is due largely to the relatively low concentration of binder included in the formulation, along with the evaporation of the carrier solvent and the segregation of the liquid component to the lower levels of the stripe during drying. Interestingly the asdeposited stripes prepared from the paste showed some conductivity before laser processing, with a typical resistivity of around $0.025 \Omega \mathrm{cm}$, over 10,000 times that of bulk copper.

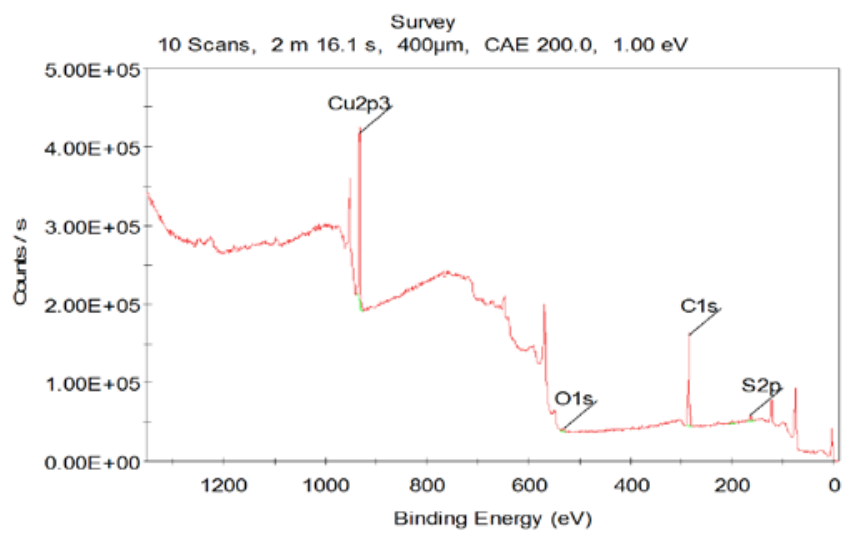

Fig. 3. XPS survey spectrum of the pre-treated copper powder.

\section{Laser Processing of Copper Tracks}

Different laser process parameters were used to treat the copper stripes to create electrically conductive tracks. Examples of the structures of the tracks obtained using the same laser scanning pattern, but different laser scanning speed and laser power, are shown in Fig. 5. Fig. 5(a) shows an overview of a $2 \mathrm{~mm}$ wide printed copper paste track after laser treatment at relatively low power. The track maintained its printed shape, but there was little evidence of any change in its microstructure from the as-deposited material, as can be seen in the higher magnification image of Fig. 5(b). This was due to the relatively low laser energy delivered to the copper. However, despite this, the track showed a significantly lower resistivity, compared to the original stripe, of $4 \times 10^{-4} \Omega \mathrm{cm}$, indicating a significant improvement in the connections between the powder particles.

With increasing laser power at the same scanning speed, changes in the structure of the tracks could be observed, with melted regions of copper identifiable on the surface of the tracks (Fig. 5(c) and (d)) that increased in area with increasing

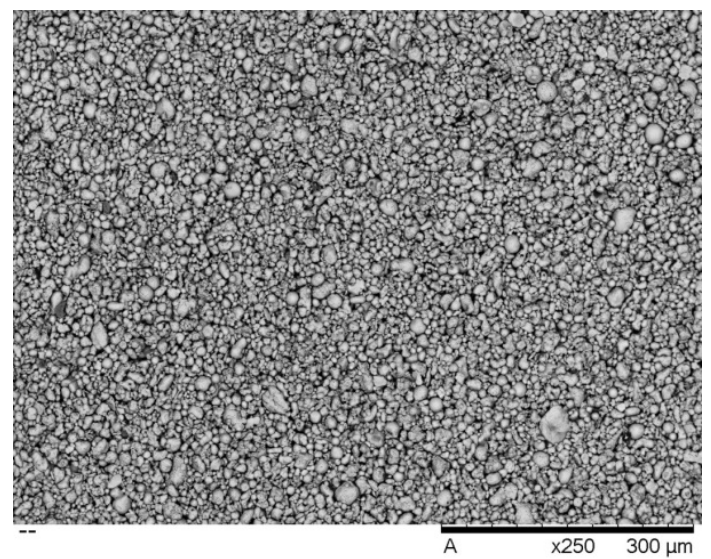

(a)

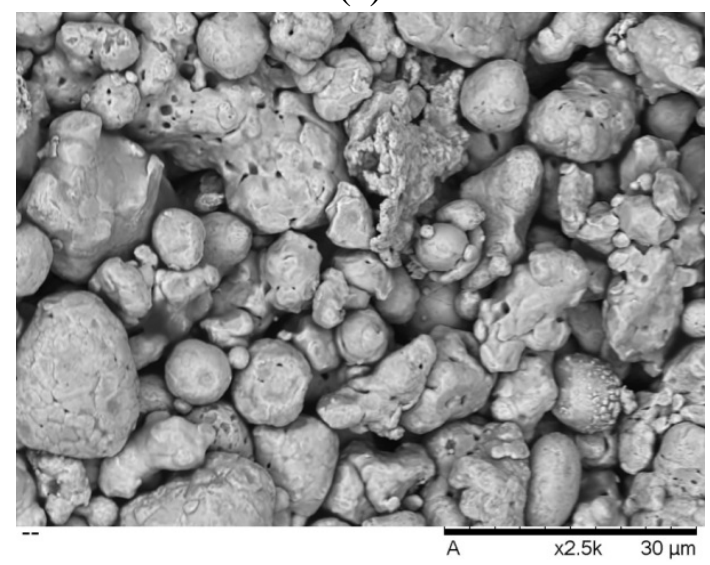

(b)

Fig. 4. SEM images of as-deposited printed tracks of copper paste; (a) low and (b) high, magnification images.

laser power. This change in laser power also led to further reduction in the resistivity of the tracks to values of around 1 to $3 \times 10^{-4} \Omega \mathrm{cm}$, about 100 times that of high purity copper and comparable to values obtained with silver filled electrically conductive adhesives [16].

Processing of loose copper powder deposited within the pre-machined grooves showed similar behaviour to that seen in the paste samples, with lower levels of laser power leading to little or no change in the powder structure. Fig. 5(e) shows an overview of a laser processed powder track where areas of partially melted copper can be seen on the top surface. Alongside the track, areas of loose powder were present as a result of the material left behind during the original groove filling process. Again, although the microstructure of the track shown in Fig. 5(e) appeared to be largely unchanged from the dry powder condition, it displayed a measurable resistance equating to a resistivity of approximately $2.5 \times 10^{-4} \Omega \mathrm{cm}$. The effect of a combined increase in scanning speed and laser power level is shown in Fig. 5(f). Increasing the scanning speed would be expected to reduce the amount of energy absorbed; however the larger melted areas that can be clearly seen in this image indicate more significant heating which is due to the increase in power level. This highlights the complex relationship between the process parameters. Additional process development led to structures such as those shown in Fig. 6, which demonstrated a further reduction in resistivity to $5 \times 10^{-5} \Omega \mathrm{cm}$, close to 30 times that of bulk copper.

$3201618^{\text {th }}$ Electronics Packaging Technology Conference 


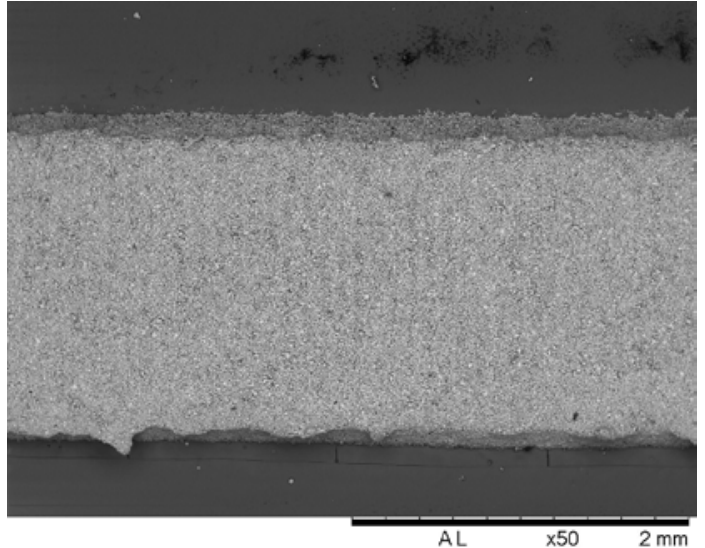

(a)

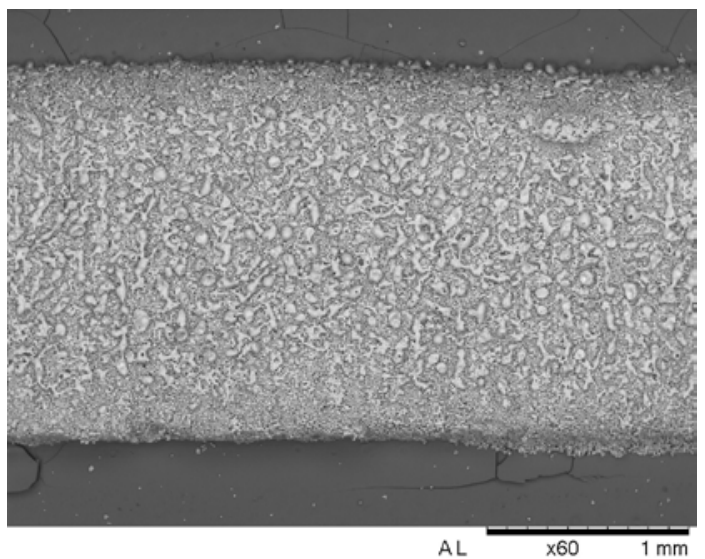

(c)

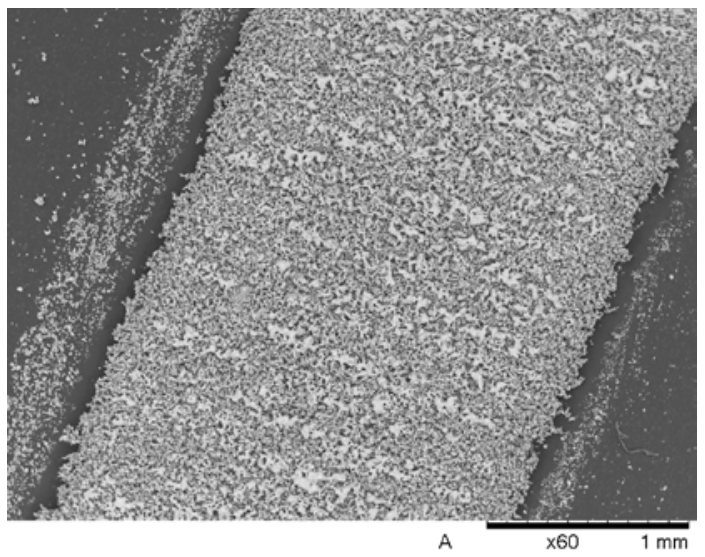

(e)

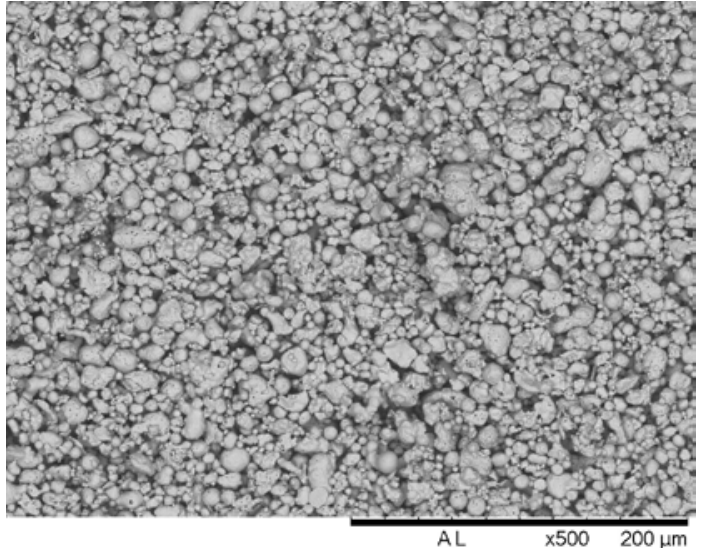

(b)

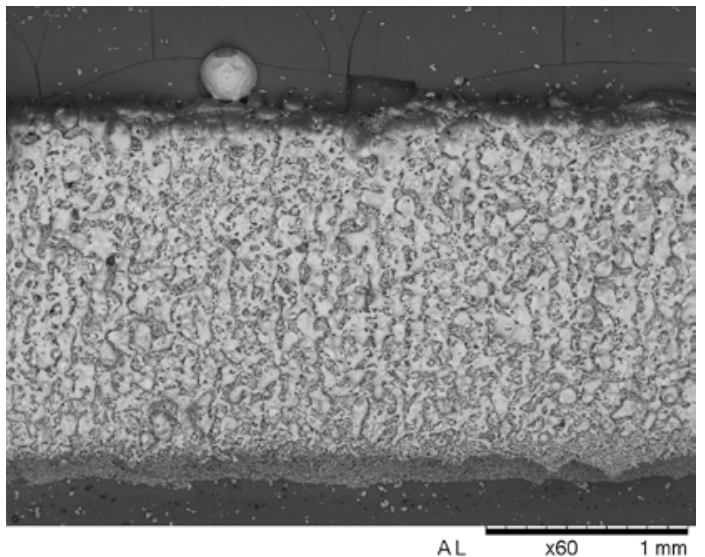

(d)

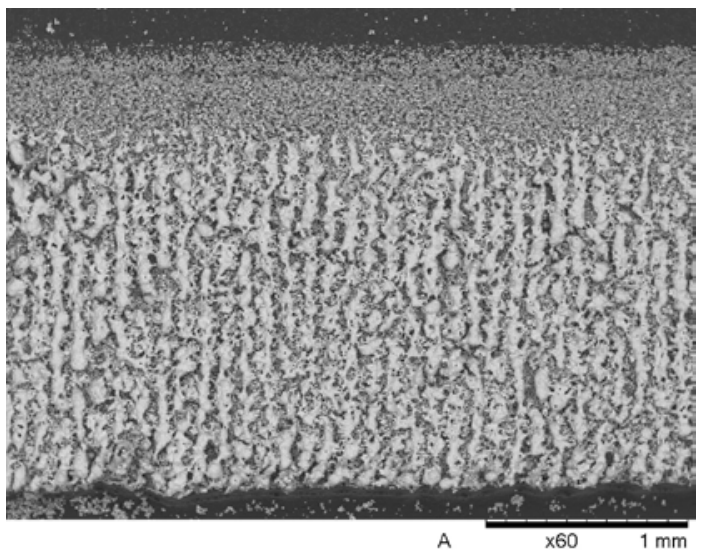

(f)

Fig. 5. SEM images of tracks processed with different laser output power and laser translation speed. Paste samples: (a, b) $16 \mathrm{~W}, 400 \mathrm{~mm} \mathrm{~s}^{-1}$ ((a) low magnification; (b) high magnification); (c) $37 \mathrm{~W}, 400 \mathrm{~mm} \mathrm{~s}^{-1}$; (d) $55 \mathrm{~W}, 400 \mathrm{~mm} \mathrm{~s}^{-1}$. Powder

samples deposited within grooves machined in an acrylic substrate: (e) $16 \mathrm{~W}, 200 \mathrm{~mm} \mathrm{~s}^{-1}$; (f) $37 \mathrm{~W}, 400 \mathrm{~mm} \mathrm{~s}^{-1}$.

As described earlier, as the diameter of the laser spot was only $0.3 \mathrm{~mm}$, it was not possible to scan over the entire track surface in a single pass and therefore the laser had to be translated across the track in a pattern of lines. Different directions and spacing of the lines were investigated. Several of the images in Fig. 5 show clearly the scan path taken by the laser, which was perpendicular to the track direction and yielded melted regions that appeared as lines running across the track. By considering the laser scanning speed and the scan pattern, the overall process speed in the direction of the track could be estimated (not including any delays at the start and end of the scanned lines). The line spacing in the examples shown here was $0.1 \mathrm{~mm}$ and, with $2 \mathrm{~mm}$ wide copper tracks and a laser translation speed of $400 \mathrm{~mm} \mathrm{~s}^{-1}$, the overall speed of processing along the length of the copper tracks was therefore approximately $20 \mathrm{~mm} \mathrm{~s}^{-1}$.

\section{Copper Track Structure}

In addition to changes in the resistivity of the tracks after laser processing many tracks also showed a significant increase in structural integrity, no longer behaving as loose 


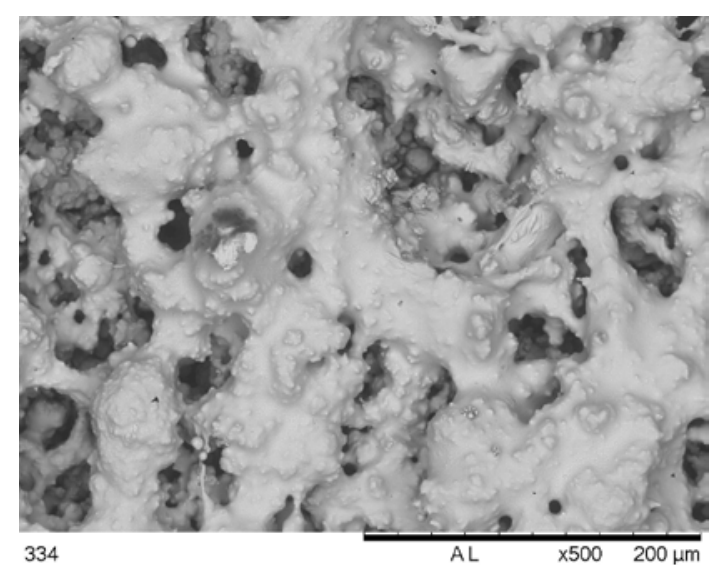

(a)

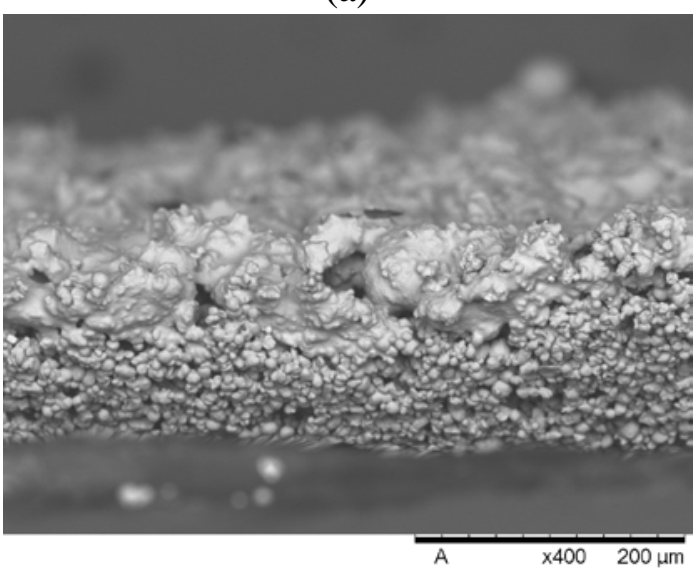

(b)

Fig. 6. SEM images of a copper track prepared from powder after detachment from the substrate. Laser processed at $27 \mathrm{~W}, 400 \mathrm{~mm} \mathrm{~s}^{-1}$ with three passes of the laser: (a) overview; (b) side view image.

powder, but becoming strong enough to be removed from the surface. This sometimes occurred spontaneously, which is believed to be due to internal stresses developed within the track, which led to deformation and separation from the substrate. Tracks made from both dry powder and from paste displayed this behaviour leaving behind a clear substrate surface and enabling the structure of the detached track to be investigated by SEM. Fig. 6 shows a track prepared by laser processing dry powder previously deposited into a groove in the acrylic substrate that had subsequently separated from the surface. The top surface (Fig. 6(a)) displayed a largely melted structure, but on closer inspection of the areas between the melted regions, individual particles of copper could be seen with a very similar structure to that of the original powder. Observation of the side of the track (Fig. 6(b)) also revealed that the top surface had a different structure consisting of large agglomerates of material that appeared to be regions of the melted powder. Below this, the copper appeared to maintain the original particle size and structure, but had become detached from the surface as a solid article, rather than as the original loose powder, indicating that the particles were now joined together. More investigation is needed to understand in detail the structure of the lower layers of the tracks and the interactions between the particles that are induced by the laser treatment. The significant reduction in track resistivity that was noted following laser processing would indicate that these lower layers provide a substantial part of the electrical conductivity and therefore would indicate a network of particle-to-particle contacts. This is supported by the observation that even where tracks show no melting (e.g. Fig. 5(a)) that conductivity is still achieved. Furthermore, even when there is significant surface melting (Fig. 5(f)) the melted lines that run perpendicular to the tracks are largely unconnected to each other and are therefore unlikely to make a significant contribution to the conductivity along the track direction.

Narrower structures were also investigated, for which an example is presented in Fig. 7. Here a feature approximately $0.75 \mathrm{~mm}$ wide has been created using dry powder deposited in a groove previously machined in acrylic. Again, with appropriate laser processing parameters, a melted structure was obtained on the top surface with a powder like structure below. Work is underway to reduce the feature sizes further and to determine the process limits.

\section{Substrate Condition}

When paste was printed onto a glass substrate and laser processed, some evidence of cracking of the glass surface on either side of the track could be seen (e.g. Fig. 5(c) and (d)). This was partly caused by misalignment between the laser path pattern and the printed track leading to the laser impinging on the glass surface and therefore causing sudden heating and subsequent rapid cooling that resulted in cracking.

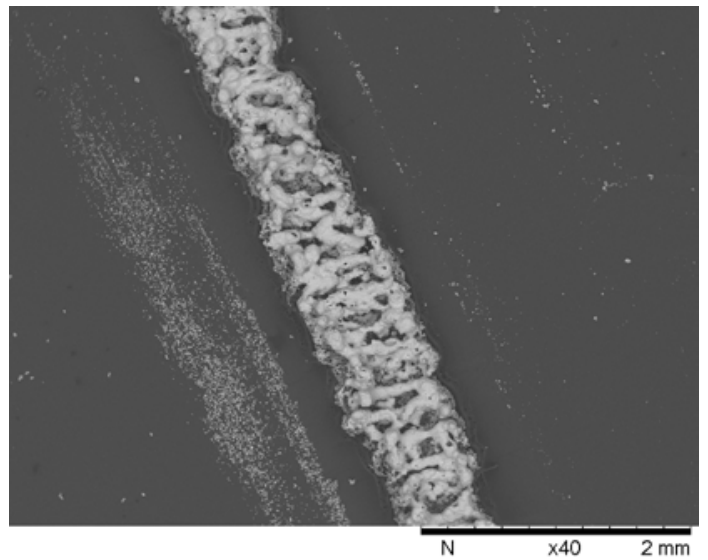

(a)

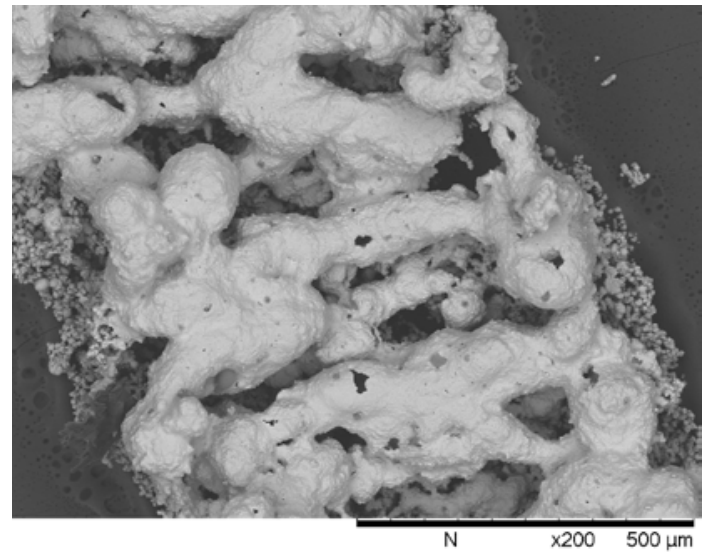

(b)

Fig. 7. SEM images of a laser processed powder track in a narrow groove on an acrylic substrate: (a) low magnification and (b) high magnification images. 
For polymer substrates, damage to the substrate at the sides of the powder tracks deposited into grooves could be seen when the laser path strayed beyond the track surface. Fig. 5(f) shows an example where the beam was misaligned with the track leading to an area (seen towards the top of the image) that was unheated, while on the opposite side of the track, the beam traversed onto the polymer substrate. Fig. 8 shows a profile of the groove machined in the acrylic substrate before filling with the copper powder and the profile of a groove after laser processing and removal of the track. The original groove had a regular profile with a flat base approximately $250 \mu \mathrm{m}$ deep. It is apparent that the shape of the base of the groove remained largely the same after laser treatment of the copper powder, indicating no significant damage to the temperature sensitive polymer material. In contrast, where the beam moved off the copper powder due to misalignment on either side of the track, the profile shows some change in shape. There is an additional trough on the right hand side where the laser readily removed acrylic material and on the left the profile is steeper. More work is underway to investigate the level of damage to the substrate and how different materials are affected.

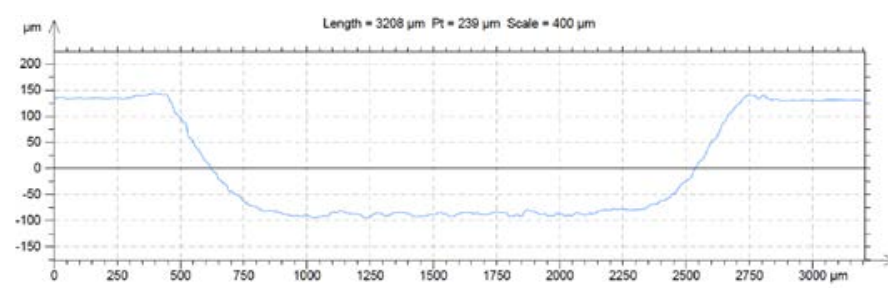

(a)

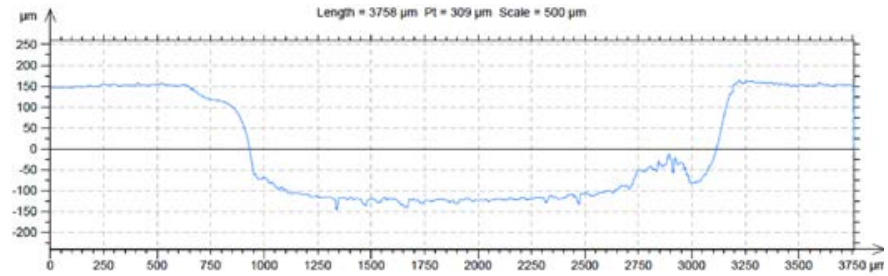

(b)

Fig. 8. Line profiles across grooves machined in acrylic: (a) before filling with copper; (b) after laser processing copper within the groove and removing the track.

\section{Conclusions}

A $\mathrm{CO}_{2}$ laser was used to thermally process copper powder to create electrically conductive tracks on polymer and glass substrates. The copper powder was first pre-treated to remove the native oxide layer and coated with a self-assembled monolayer to restrict re-oxidation during storage and deposition before laser processing. Copper powders were successfully processed in air with a variety of power levels, laser translation speeds and different pathways across the surface. It was found that with low laser power that the copper appeared to be unmelted, but this still led to a significant reduction in resistivity compared to the as-deposited material. In addition, the tracks no longer behaved as loose powder indicating some bonding between the particles. With higher laser energy density, melted regions on the top of the tracks were observed with a particulate structure remaining below this. This led to a further reduction in resistivity with the best samples showing levels that were around 30 times that of bulk copper. Investigation of the acrylic substrate after laser processing showed damage to areas directly exposed to the laser, but no obvious damage to regions underneath the copper. Further work is underway to optimize the process, including reducing the dimensions of the features and understanding the adhesion of the copper to the substrate, such that the technology can be integrated successfully into the fabrication of embedded electronic devices.

\section{References}

1. Scandurra, A., et al, "Low-temperature sintered conductive silver patterns obtained by inkjet printing for plastic electronics," Surf. Interface Anal., vol. 42, no. 6-7 (2010), pp. 1163-1167.

2. Perelaer, J., et al, "Inkjet-printed silver tracks: low temperature curing and thermal stability investigation," $J$. Mater. Chem., vol. 18, no. 27 (2008), p. 3209.

3. Santos, E.C., et al, "Rapid manufacturing of metal components by laser forming," Int. J. Mach. Tools Manuf., vol. 46, no. 12-13 (2006), pp. 1459-1468.

4. Kruth, J. P., et al, "Consolidation phenomena in laser and powder-bed based layered manufacturing," CIRP Ann. Manuf. Technol., vol. 56, no. 2 (2007), pp. 730-759.

5. Yadroitsev, I., et al, "Single track formation in selective laser melting of metal powders," J. Mater. Process. Technol., vol. 210, no. 12 (2010), pp. 1624-1631.

6. Yadroitsev, I. \& Smurov, I., "Selective laser melting technology: From the single laser melted track stability to 3D parts of complex shape,” Phys. Procedia, vol. 5, part 2 (2010), pp. 551-560.

7. Hidai, H. \& Tokura, H., "Direct laser writing of aluminum and copper on glass surfaces from metal powder," Appl. Surf. Sci., vol. 174, no. 2 (2001), pp. 118-124.

8. Tang, Y., et al, "Direct laser sintering of a copper-based alloy for creating three-dimensional metal parts," J. Mater. Process. Technol., vol. 140, no. 1-3 (2003), pp. 368-372.

9. Zhu, H.H., et al, "Development and characterisation of direct laser sintering Cu-based metal powder," J. Mater. Process. Technol., vol. 140, no. 1-3 (2003), pp. 314-317.

10. Zenou, M., et al, "Laser sintering of copper nanoparticles," J. Phys. D. Appl. Phys., vol. 47, no. 2 (2014), p. 25501.

11. Shishkovsky, I., et al, "Low-dose laser sintering of $\mathrm{Cu}$ nanoparticles on the ceramic substrate during ink-jet interconnection," Fundam. Laser-Assisted Micro- Nanotechnologies Conf., vol. 9065, no. 846 (2013), p. 90650I.

12. Laibinis, P.E. \& Whitesides, G.M., "Self-Assembled Monolayers of n-alkanethiolates on copper are barrier films that protect the metal against oxidation by air," $J$. Am. Chem. Soc., vol. 23, no. 114 (1992), pp. 9022-9028.

13. Bohren, C.F. \& Huffman, D.R., Absorption and Scattering of Light by Small Particles, John Wiley \& Sons, (2008).

14. Brown, M.S. \& Arnold, C.B. in Laser Precision Microfabrication, Springer Ser. Mater. Sci., vol. 135, (2010), pp. 91-120.

15. Kruth, J.P., et al, "Lasers and materials in selective laser sintering,” Assem. Autom., vol. 23, no. 4 (2003), pp. 357371.

16. Yim, M.J. \& Paik, K.W., "Review of Electrically Conductive Adhesive Technologies for Electronic Packaging," Electron. Mater. Lett., vol. 2, no. 3 (2006), pp. 183-194. 